\title{
Performance stabilization of CdTe PV modules using bias and light
}

\author{
Timothy J Silverman, Michael G. Deceglie, Bill Marion, Sarah R. Kurtz \\ National Renewable Energy Laboratory, Golden, Colorado, 80401, United States
}

\begin{abstract}
Reversible performance changes due to light exposure frustrate repeatable performance measurements on CdTe PV modules. It is common to use extended light-exposure to ensure that measurements are representative of outdoor performance. We quantify the extent to which such a light-exposed state depends on module temperature and consider bias in the dark to aid in stabilization. We evaluate the use of dark forward bias to bring about a performance state equivalent to that obtained with light exposure, and to maintain a light-exposed state prior to STC performance measurement. Our results indicate that the most promising method for measuring a light-exposed state is to use light exposure at controlled temperature followed by prompt STC measurement with a repeatable time interval between exposure and the STC measurement.
\end{abstract}

Index Terms-CdTe, thin-film, module, performance, stabilization, light soak, metastability, transient

\section{BACKGROUND AND INTRODUCTION}

Measuring the performance of a PV module under standard test conditions (STC) is useful for predicting outdoor performance of new modules and for quantifying performance changes due to accelerated testing or outdoor exposure. In the dominant thin-film PV technologies, namely CdTe, CIGS and a-Si, obtaining an STC performance measurement is frustrated by reversible, metastable changes in performance on time scales ranging from seconds to several days and in magnitudes on the order of $15 \%$ [1]. Furthermore, these metastable changes can be excited or relaxed by accelerated testing, and if not carefully controlled can be confused with permanent degradations associated with the accelerated tests.

Due to the existence of these metastable changes, thinfilm modules often have a range of electrical performance states dictated by the time-, temperature-, voltage- and lightdependent chemical and material properties of the solar cells. In order to frame our discussion it is useful to define several terms associated with these states. We use the term "lightexposed state" to refer to an electrical performance state that is representative of that achieved under typical outdoor operation. As we will show, this light-exposed state is not necessarily unique and can depend on the operating temperature of the module. Also note that the light-exposed state could, in principle, be brought about with an appropriate procedure in the absence of illumination by, for example, applying electrical bias to the module. We adopt the term "repeatable state" to refer to an electrical performance state that can be repeatably achieved through means of some procedure, regardless of how representative the state is of operation outdoors. We define "stabilization procedure" as any procedure that is intended to bring about either a light-exposed state or a repeatable state.

Stabilization procedures often involve prolonged exposure to natural or artificial sunlight, sometimes called light soaking. Indeed, the only currently standardized method of stabilization is the method in IEC 61646, the type qualification test for thinfilm PV modules [2]. This method requires repeated exposure to sunlight in increments of $43 \mathrm{kWh} / \mathrm{m}^{2}$ until successive power measurements differ by $<2 \%$. In this work we performed a variety of experiments on commercial $\mathrm{CdTe}$ modules to understand the efficacy of different stabilization procedures, paying particular attention to whether they are successful in enabling STC measurement of the light-exposed state. The experiments are described in detail in Section III while our results and their implications are discussed in Section III. We discuss the limitations of the various stabilization procedures, including light exposure, and conclude with recommendations for making repeatable STC performance measurements representative of outdoor performance.

In one experiment, described in Sections II-A and III-A, we consider how the temperature during light exposure affects the stabilized state, highlighting that the light-stabilized state of a module depends on exposure conditions. We also consider the use of forward bias in the dark to both bring about the light-stabilized state and to stabilize performance for the time interval between light-exposure and measurement.

The use of forward bias in the dark has been shown in CIGS modules to maintain the light-exposed state [3]. This addresses the challenge that the delay between light exposure and indoor performance measurement can result in the partial or complete relaxation of a module to its unexposed electrical performance state. In sections II-B and III-B, we describe experiments designed to evaluate this approach for $\mathrm{CdTe}$ modules and present their results.

Forward bias in the dark has also been considered as a means of bringing about the light-stabilized performance state; the use of forward bias at elevated temperature (BET) without illumination has been proposed in the CdTe-specific section of a draft of the third edition of IEC 61215. In this study we tested a version of the proposed BET method, comparing the results to those obtained using ordinary light exposure. Interestingly, we found that modules which responded differently from each other to light exposure responded in the same way to BET, suggesting that while it may produce repeatable results, BET does not reliably bring about an outdoor-relevant light-exposed state. This highlights the challenge that metastable module behavior can be dictated by a number of different physical mechanisms, and that the particular mix of these mechanisms that any one module exhibits may affect its response to different techniques. These experiments and their results are described in sections II-C and III-C

\section{METHOD}

We used full-size, commercial CdTe modules from two manufacturers, referred to as "A-type" specimens and "B- 
type" specimens. A-type specimens had been removed from outdoor deployment and stored in darkness for $>90 \mathrm{~d}$. B-type specimens were previously unexposed.

For continuous light exposure tests, we used a lightexposure chamber with class BBA light at $1000 \mathrm{~W} / \mathrm{m}^{2}$ irradiance. The mean of the measured back-of-module temperatures was controlled using forced convection. The instrument measured light I-V characteristics every (2 to 5) min and each module was biased at $P_{\mathrm{mp}}$ between measurements. In this work, $P_{\mathrm{mp}}$ measurements from this instrument were corrected using "self-irradiance" by multiplying by $I_{\mathrm{sc}}^{\text {nominal }} / I_{\mathrm{sc}}$. No temperature correction was applied to these measurements.

For individual light I-V measurements, we used a long-pulse solar simulator with class AAA light. Using the measured back-of-module temperature, we temperature-corrected measurements from this instrument using the technique described in Section II-D No self-irradiance correction was applied to these measurements.

Elevated-temperature tests were performed in a temperaturecontrolled environmental chamber. Humidity control was not used, resulting in relative humidity of $<10 \%$ during tests at $55^{\circ} \mathrm{C}$ and $<3 \%$ during tests at $85^{\circ} \mathrm{C}$.

\section{A. Temperature-dependent stabilization}

In order to understand how module temperature affects the light-exposed state of the modules, we exposed the modules in the light-exposure chamber under a series of different back-ofmodule temperature setpoints. The modules were exposed to simulated sunlight at $1000 \mathrm{~W} / \mathrm{m}^{2}$ until the $P_{\mathrm{mp}}$ was changing $<1 \% /(24 \mathrm{~h})$. After this condition was met, the chamber was turned off for (5-10) min to enable reprogramming, then restarted. The first $2 \mathrm{~h}$ of the subsequent step were carried out at the same temperature as the preceding step, then the chamber setpoint changed to the new temperature for the remainder of the step. This was carried out for the following sequence of temperature setpoints: $55^{\circ} \mathrm{C}, 62.5^{\circ} \mathrm{C}$, $70^{\circ} \mathrm{C}, 62.5^{\circ} \mathrm{C}, 55^{\circ} \mathrm{C}$. Near the end of the first step of this sequence, we carried out the temperature coefficient procedure described in Section II-D.

\section{B. Post-light-exposure stabilization}

We performed a series of experiments to study whether the application of forward bias to modules between light exposure and indoor light I-V measurements aids in stabilizing the module in its light-exposed state for the indoor measurement. Modules were exposed in the light-exposure chamber with a back-of-module setpoint temperature of $55^{\circ} \mathrm{C}$ until their $P_{\mathrm{mp}}$ was changing $<1 \% /(24 \mathrm{~h})$. When the module performance was stable, the temperature coefficient measurement described in Section II-D was performed. In the case of the A-type modules, part of the stabilizing light-exposure was the experiment described in Section II-A, but the last portion was performed at $55^{\circ} \mathrm{C}$

On a clear day, and once the modules' performance was stable in the light-exposure chamber, they were relocated to an outdoor test rack located just outside the long-pulse simulator laboratory. On this outdoor rack, the modules were exposed to natural sunlight and maximum power-point tracked with I-V curves collected at 15-min intervals. The transition time between the light-exposure chamber and the outdoor lightexposure was less than $15 \mathrm{~min}$ and the modules were exposed on the outdoor rack for at least $1.25 \mathrm{~h}$. Immediately following this outdoor exposure, we collected a series of pulses on the long-pulse simulator. A series of five measurements were made at one-minute intervals followed by measurements approximately once every $20 \mathrm{~min}$ for a total of $2 \mathrm{~h}$. We then returned the modules to the light-exposure chamber at $1000 \mathrm{~W} / \mathrm{m}^{2}$ and $55^{\circ} \mathrm{C}$ for (12-48) $\mathrm{h}$ before repeating the outdoor-exposure and long-pulse simulator measurement procedure. On this repetition, the modules were forward biased with a DC power supply between light I-V measurements in constant current mode. The current setpoint was that which the modules passed when biased to $90 \%$ of their expected STC $V_{\text {oc }}$ in the dark immediately upon being brought indoors.

\section{Bias-only stabilization}

We performed two different experiments to investigate the efficacy of applying bias to modules in the absence of light in order to bring about a light-exposed state. In the "biaslight" experiment the modules were first subjected to forward bias at elevated temperature (BET), followed immediately by light exposure. In the "light-anneal-bias" experiment, the modules were first exposed to light, then annealed at elevated temperature in darkness to allow modules to relax back to their dark state, and finally subjected to BET.

The BET procedure used in this work was adapted from that proposed in the draft version of edition 3 of IEC 61215. Briefly, the method proposed in the draft standard is:

1) Collect a light $I-V$ curve

2) Place the test article in a dark test chamber and bring the air temperature to $85^{\circ} \mathrm{C}$ at $\geq 180^{\circ} \mathrm{C} / \mathrm{h}$

3) Apply a fixed-voltage bias of (0.9 to 1.0$) \times V_{\text {oc }}$ to the test article for $1.5 \mathrm{~h}$

4) Discontinue bias and return the chamber to room temperature at $\leq-180^{\circ} \mathrm{C} / \mathrm{h}$

5) Collect a light I-V curve

6) Repeat steps 2 through 5 until three successive measurements result in $\frac{P_{\max }-P_{\min }}{P_{\text {mean }}}<0.02$

In this work, we altered the method in two ways: to reduce stress on the modules, we changed the bias in step 3 to $V_{\mathrm{mp}}$, which was $60 \%-75 \%$ of $V_{\mathrm{oc}}$. And to complete the experiment in a timely manner we used $\frac{P_{\max }-P_{\min }}{P_{\operatorname{mean}}}<0.04$ in step 6 . In our facility a maximum of three bias steps can be performed per workday. We stored the test articles at room temperature between shifts of bias testing. The I-V curves in this segment of the test were measured on a long-pulse solar simulator at $25^{\circ} \mathrm{C}$.

Prior to the bias-light experiment the modules were exposed to continuous artificial sunlight in the light-exposure chamber at $1000 \mathrm{~W} / \mathrm{m}^{2}$ with back-of-module temperature of $\sim 55^{\circ} \mathrm{C}$ for $>100 \mathrm{~h}$. The specimens were then annealed in darkness at $85^{\circ} \mathrm{C}$ for $100 \mathrm{~h}$. To begin the bias-light experiment, the modules underwent the BET procedure until the stopping criterion was met, at which point we placed it the lightexposure chamber at $1000 \mathrm{~W} / \mathrm{m}^{2}$ illumination with back-ofmodule temperature at $\sim 55^{\circ} \mathrm{C}$.

For the light-anneal-bias experiment, the modules were first exposed in the light-exposure chamber at $1000 \mathrm{~W} / \mathrm{m}^{2}$ 
with back-of-module temperature of $\sim 55^{\circ} \mathrm{C}$ until the $P_{\mathrm{mp}}$ was changing $<1 \% /(24 \mathrm{~h})$. Once the modules were stable the temperature coefficient measurement described in Section II-D was carried out. For the purposes of the post-light-exposure stabilization experiment, the modules then underwent the steps described in Section II-B. The light-anneal-bias experiment then proceeded with a $168-\mathrm{h}$ anneal in the dark at $55^{\circ} \mathrm{C}$. We then applied the BET procedure and collected the associated I-V curves.

\section{Temperature correction}

We measured module-specific temperature coefficients in the light-exposure chamber after $\geq 100 \mathrm{~h}$ of light exposure. The temperature was perturbed by $\pm 2{ }^{\circ} \mathrm{C}$ for $\geq 1 \mathrm{~h}$ per step while I-V measurements took place. We used the resulting data set to calculate absolute temperature coefficients (for instance the $P_{\mathrm{mp}}$ temperature coefficient in $\mathrm{W} / \mathrm{K}$ ) by minimizing the standard deviation of the distribution of temperature-corrected values for the entire period. These temperature coefficients are used to apply additive temperature corrections to measurements made on the long-pulse simulator.

\section{RESULTS AND DISCUSSION}

\section{A. Temperature-dependent stabilization}

The stepped-temperature light-exposure experiment was performed to quantify how the light-exposed state of the modules depends on operating temperature. The results are shown in Figure 1. In this figure, $P_{\mathrm{mp}}$ is not temperature-corrected and its relationship with temperature is superficially ordinary: $P_{\mathrm{mp}}$ is lower during the higher-temperature steps. However, at the beginning of each step is a transient change in $P_{\mathrm{mp}}$ that is many times slower than the change in module temperature and is in the opposite direction from the change that would be associated with a lag in module temperature. That is, the time series appears to be affected by two changes, the usual temperature dependence of the power which is captured by the temperature coefficient, and also a restabilization as the module reaches a new electrical performance state. This restabilization reverses in the downward temperature steps, supporting the interpretation that the module is reaching a different electrical performance state depending on its temperature. This change is approximately $(1-2) \%$ for each $7.5^{\circ} \mathrm{C}$ temperature step. We verified that the apparent restabilization was not an artifact of the method by repeating the procedure on a crystalline Si module. The Si module exhibited only the usual temperature dependence associated with the temperature coefficient.

The temperature shown in Figure 1 is back-of-module temperature. The relationship between back-of-module temperature and the temperature of the PV junction depends on many factors, including prevailing outdoor conditions or the design of light-exposure equipment. These factors must be considered when comparing $P_{\mathrm{mp}}$ measurements obtained after stabilization outdoors or in different types of light-exposure chambers.

The size of the re-stabilization is of less interest here than

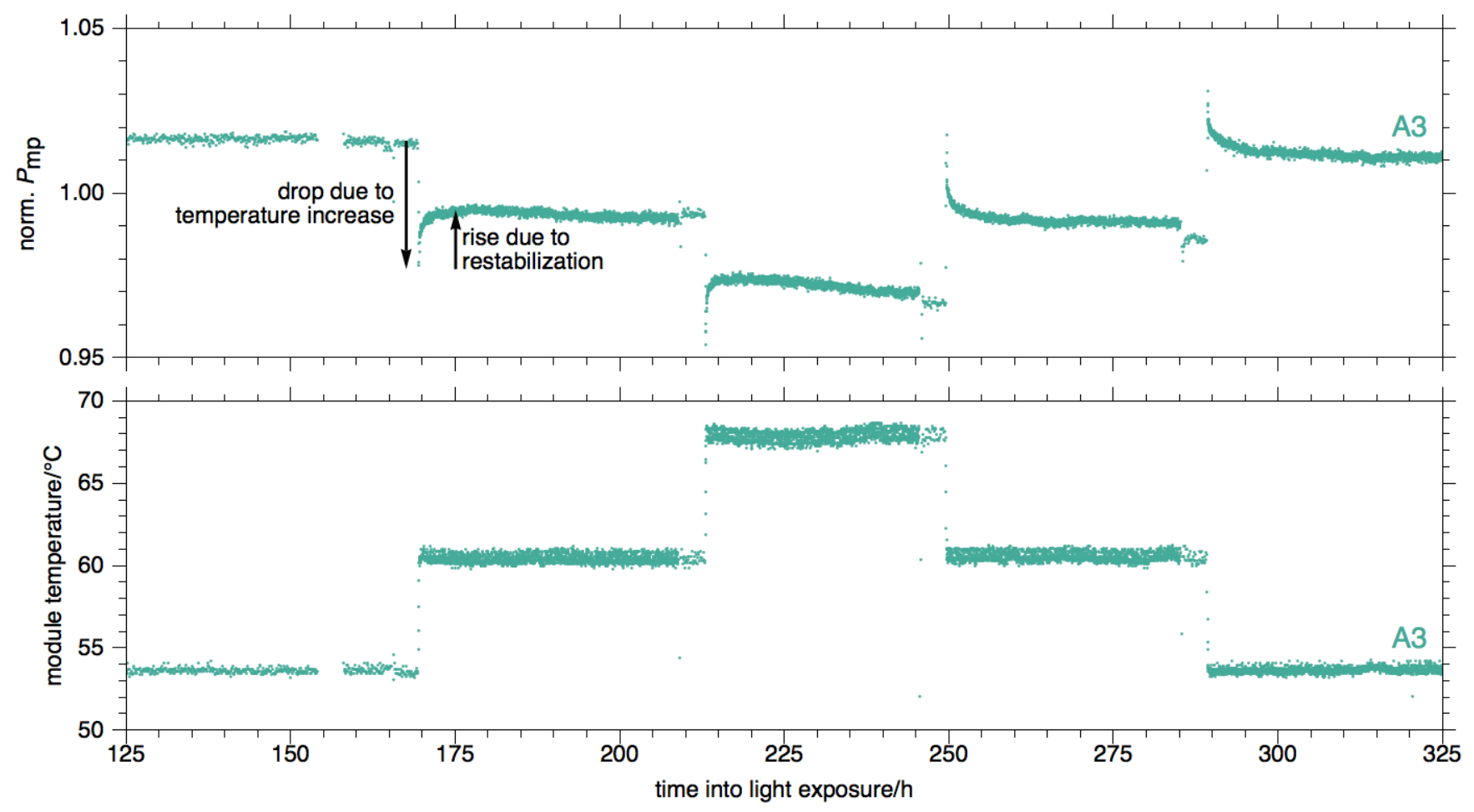

Fig. 1. $\quad P_{\mathrm{mp}}$ and temperature from the stepped-temperature light exposure test are shown for module A3. $P_{\mathrm{mp}}$ is normalized to the median value for the entire test. The transient changes in $P_{\mathrm{mp}}$ last much longer than module temperature transients and are in the opposite direction from $P_{\mathrm{mp}}$ changes due only to temperature. Note that the plot starts at $125 \mathrm{~h}$ into the test. 


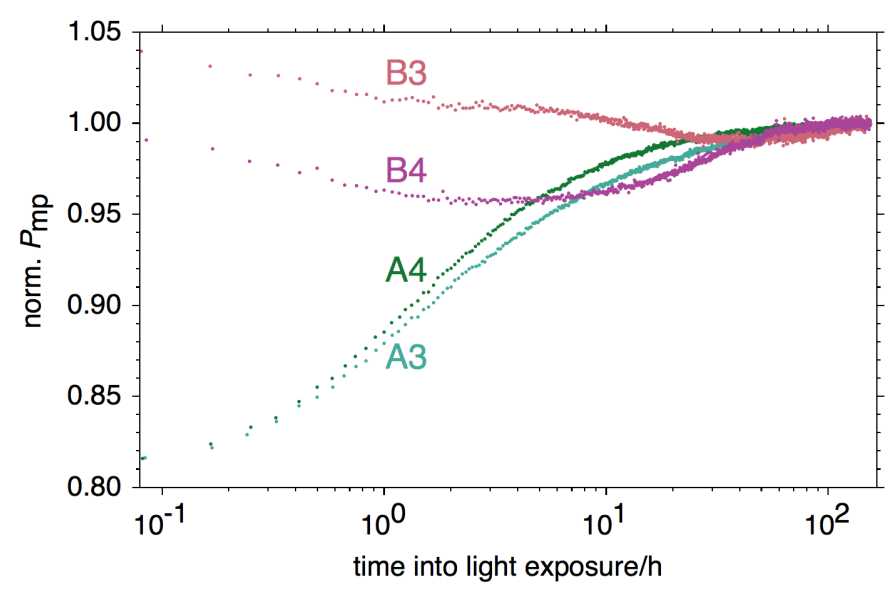

Fig. 2. The light exposure step of the post-light-exposure stabilization test caused $P_{\mathrm{mp}}$ changes in both directions and up to $16 \%$ in magnitude. These data are normalized to the final measurement and are labeled with the module identifier.

its existence, because different modules are expected to exhibit different magnitudes of temperature-dependent restabilization. This result shows that a module's light-exposed state is generally dependent on operating temperature during exposure. For repeatable stabilization using light exposure, we recommend that equipment be used where the module temperature can be kept the same for each light exposure.

\section{B. Post-light-exposure stabilization}

The post-light-exposure relaxation experiments were performed to evaluate whether the application of forward bias is an effective means to maintain performance in the lightexposed state between light exposure and STC measurement. The first step in these experiments was light exposure, the results from which are shown in Figure 2. Module type A shows an increase in $P_{\mathrm{mp}}$ of up to $16 \%$. Module type B shows two different behaviors upon light exposure: B3 declines in performance and B4 improves after a short initial decrease. All of the observed $P_{\mathrm{mp}}$ changes were driven by changes in $V_{\text {oc }}$ and $F F$. The variety of observed behaviors highlights the difficulty of finding one-size-fits-all testing methods for thinfilm modules.

The effect of room-temperature forward bias between light exposure and flash I-V measurements is illustrated in Figure 3 Without bias, $P_{\mathrm{mp}}$ changes by up to $3 \%$ in the first two hours, with some modules improving and some degrading. When bias is applied before measurement, all of the modules decline in performance approximately $2 \%$ to $3 \%$.

The application of post-light-exposure bias fails to hold the modules in their light-exposed state, in contrast to what has been observed on CIGS modules [3]. Applying bias complicates the test procedure by introducing additional equipment and makes reaching a desired temperature more difficult due to self-heating. We recommend that bias not be used in an attempt to keep modules in their light-exposed state between light exposure and flash testing. To achieve repeatable
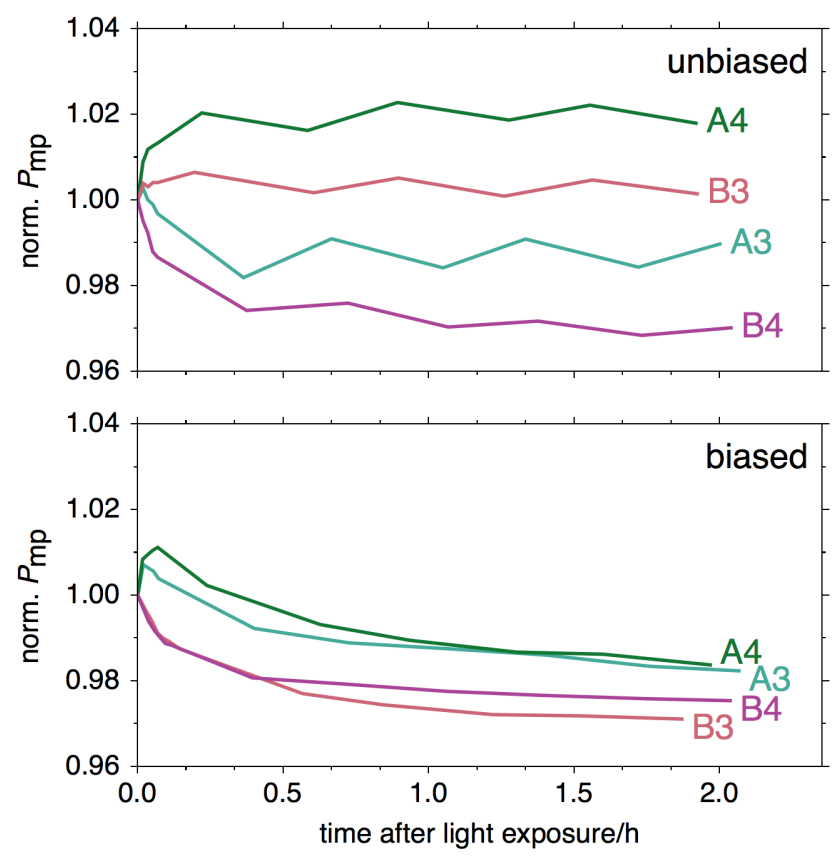

Fig. 3. Results from the unbiased relaxation and biased relaxation tests show that room-temperature forward bias fails to stabilize $P_{\mathrm{mp}}$ during the time between light exposure and flash testing. These measurements are normalized to the initial measurement. Lines are labeled with the module identifier.

measurements representative of the light-exposed state, the delay between light exposure and flash testing should be short and kept constant for each specimen. For example, using a delay of approximately 1 hour is expected to yield repeatable results. A much shorter delay than this introduces the risk of taking a measurement while performance is changing rapidly.

\section{Bias-only stabilization}

We performed two tests of the BET stabilization procedure: In the "bias-light" test, BET was immediately followed by light exposure to determine whether light would cause further changes. In the "light-anneal-bias" test, we performed the light exposure first, to determine the character of the modules' transients. A subsequent $55^{\circ} \mathrm{C}$ anneal step relaxed the modules before application of the BET procedure.

Results from the bias-light test are shown in Figure 4 Modules A1 and A2 showed a $\sim 20 \%$ increase in $P_{\mathrm{mp}}$ during BET. The change was driven mostly by a $V_{\mathrm{oc}}$ improvement, with a contribution from $F F$ increase. There was virtually no further change in performance upon light exposure. In contrast, module B2 showed virtually no performance change during BET but changed substantially upon light exposure, mostly due to $F F$ decrease. This suggests that at the end of the bias procedure the module was in a state that was different from its light-exposed state under our light-exposure conditions.

Results from the light-anneal-bias test are shown in Figure 5 The continuous light exposure data in the left pane of Figure 5 is the same as that shown in Figure 2 Modules B3 and B4 have opposite behavior when exposed to light: B4 

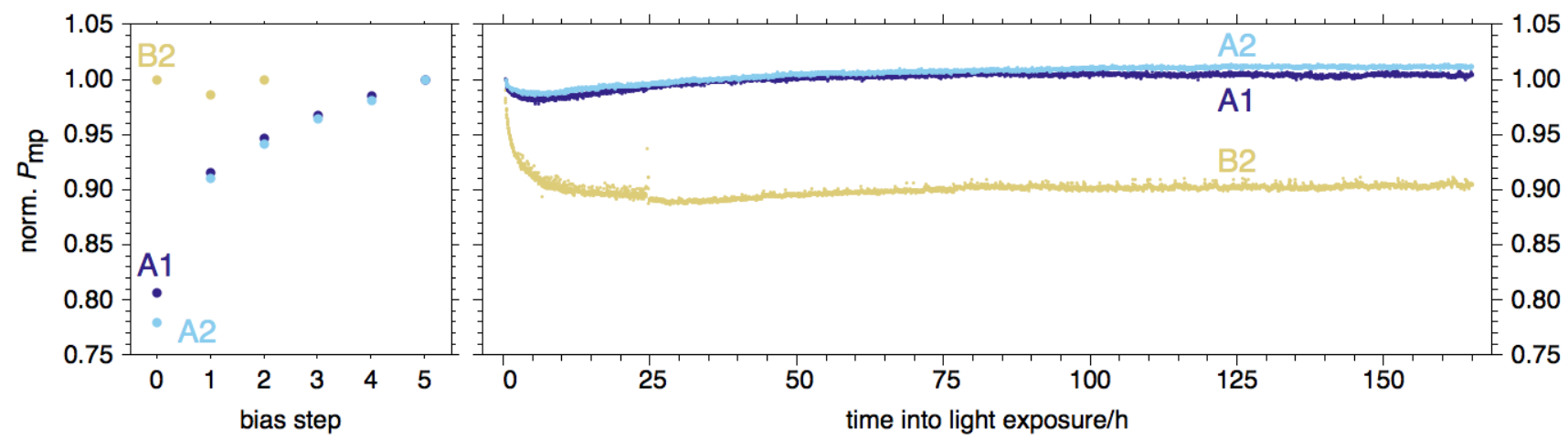

Fig. 4. Evolution of $P_{\mathrm{mp}}$ during BET (left) and continuous light exposure (right) as part of the bias-light test. Module B2 undergoes further performance changes upon light exposure, suggesting that light exposure excites phenomena that are unaffected by the BET process. $P_{\mathrm{mp}}$ is normalized to the final measurement in the left pane and to the initial measurement in the right pane. Data are labeled with the module identifier.
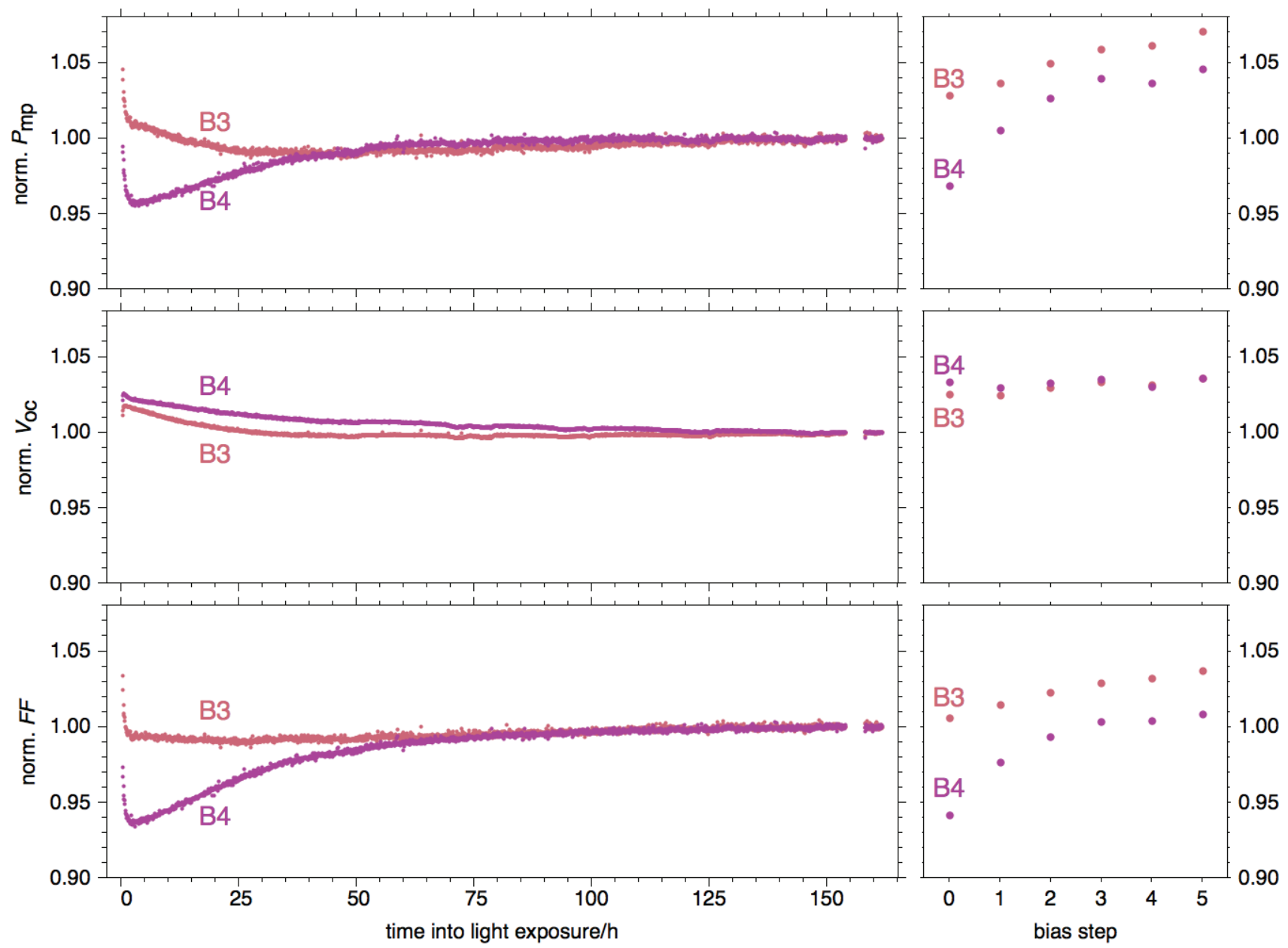

Fig. 5. Evolution of performance parameters during continuous light exposure (left) and, after annealing at $55^{\circ} \mathrm{C}$, the $\mathrm{BET}$ procedure (right) as part of the light-anneal-bias test. Although module B3 shows a $P_{\mathrm{mp}}$ decline with light exposure, its $P_{\mathrm{mp}}$ improves with application of the BET procedure. Parameters are normalized to the final measurement in the left pane and to a post-light-exposure (pre-anneal) flash test taken approximately $1 \mathrm{~h}$ after the light exposure in the right pane. The extent to which the modules recovered to their pre-light-exposure state during the anneal is reflected in the bias step 0 points in the right panes. Data are labeled with the module identifier. 
improves while B3 degrades. This difference in transient behavior is due to a substantial rise in the $F F$ of module B4 upon light exposure, while B3 had a relatively stable $F F$ during light exposure. A reversible, light-induced $F F$ improvement as seen on module B4 has been observed before [4]. Both modules showed a decrease in $V_{\text {oc }}$ with light exposure. The continuous light exposure data show a fast, $\sim 1 \mathrm{~h}$ performance change followed by a more gradual change. The anneal step returns module performance to that seen during the fast initial change, as shown in bias step 0 in Figure 5 . However, during bias stabilization, the modules both improve. That is, modules B3 and B4 move in opposite directions during light exposure but both move in the same direction during bias stabilization. We attribute this result to the modules' different $F F$ transient behavior. The results show that BET does not consistently reproduce the effects of light exposure, and the relationship between the BET induced state and the light-induced state depends on the behavior of a particular module.

Both light-exposure and BET are available as stabilization techniques for CdTe modules in the current IEC 61215 edition 3 draft. The observation that the two methods may produce different results needs to be considered. That some modules have similar response to light and bias and some do not suggests that a variety of mechanisms is present. In fact a variety of mechanisms have been proposed to explain metastable changes to CdTe cells and modules, including light-modulated energetic barriers and changing occupation of bulk traps; copper used to optimize the back contact barrier height is also widely understood to have an important role in determining performance and stability, though it is often associated with degradation rather than reversible metastability [1], [5]-[9].

Given the variety of mechanisms that is likely to be present, it is not surprising that BET and light exposure are observed to affect the modules in different ways. During BET, electron hole quasi-Fermi splitting in the CdTe absorber is similar to that achieved with photo-excitation during light exposure. However in the buffer and front contact, the quasi-Fermi levels are different in dark forward bias and in light exposure. Thus a particular product's mix of metastability mechanisms, especially those involving interfacial barrier heights, is expected to determine its responses to the two methods of stabilization. While BET may produce a repeatable state (a possibility not investigated in this work), our results suggest that it does not consistently result in a light-exposed state. Where performance measurements need to be representative of outdoor performance, we do not recommend the BET technique for stabilization.

\section{CONCLUSION}

We have shown that the performance of CdTe modules stabilized using light exposure depends on the temperature of the module during the light exposure. We further demonstrated that the application of room-temperature forward bias after light exposure fails to keep modules in their lightexposed state. Finally, we showed that the proposed elevatedtemperature dark-bias stabilization method (BET) is not equivalent to light exposure and can produce performance changes in the opposite direction of the changes caused by light exposure.

It is important to adopt a stabilization procedure to control for metastable changes to thin-film module performance. In some cases, it is desirable to reproduce the same electrical performance state that the module would achieve when deployed outdoors (the light-exposed state). In other cases repeatability alone may be sufficient. In those cases where outdoor performance is sought, our results show that lightexposure is preferable to the BET procedure studied here. It is important to note that the state achieved with light exposure is not necessarily a unique state and can depend on module operating temperature. It is thus important that lightexposure be carried out at a repeatable module temperature. Furthermore, prompt and repeatable time intervals should be adopted between light-exposure and solar simulator I-V measurements to maximize repeatability.

\section{ACKNOWLEDGMENT}

We thank Keith Emery, Steve Rummel and Allan Anderberg for performing I-V measurements on the pulsed simulator, Kent Terwilliger and Greg Perrin for performing the anneal and dark bias testing and Gary Babbitt for chamber assistance. Some of the data in this report were obtained using equipment at the Energy Systems Integration Facility (a national user facility sponsored by the U.S. DOE Office of Energy Efficiency and Renewable Energy) located at the National Renewable Energy Laboratory. This work was supported by the U.S. Department of Energy under Contract No. DE-AC3608GO28308 with the National Renewable Energy Laboratory.

\section{REFERENCES}

[1] M. Gostein and L. Dunn, "Light soaking effects on photovoltaic modules: overview and literature review," in Photovoltaic Specialists Conference (PVSC), 2011 37th IEEE. IEEE, 2011, pp. 003 126-003 131.

[2] IEC 61646, "Thin-film terrestrial photovoltaic (PV) modules: Design qualification and type approval," IEC Standard, 2008.

[3] C. Deline, A. Stokes, T. J. Silverman, S. Rummel, D. Jordan, and S. Kurtz, "Electrical bias as an alternate method for reproducible measurement of copper indium gallium diselenide (CIGS) photovoltaic modules," in SPIE Solar Energy+ Technology. International Society for Optics and Photonics, 2012, pp. 84720 G-84720G.

[4] C. Deline, J. del Cueto, D. Albin, C. Petersen, L. Tyler, and G. TamizhMani, "Transient response of cadmium telluride modules to light exposure," in Photovoltaic Specialists Conference (PVSC), 2011 37th IEEE, June 2011, pp. 003 113-003118.

[5] G. Agostinelli, E. D. Dunlop, D. L. Batzner, A. N. Tiwari, P. Nollet, M. Burgelman, and M. Kontges, "Light dependent current transport mechanisms in chalcogenide solar cells," in Photovoltaic Energy Conversion, 2003. Proceedings of 3rd World Conference on, vol. 1, 2003, pp. 356-359.

[6] M. Köntges, R. Reineke-Koch, P. Nollet, J. Beier, R. Schäffler, and J. Parisi, "Light induced changes in the electrical behavior of CdTe and $\mathrm{Cu}(\mathrm{In}, \mathrm{Ga}) \mathrm{Se}_{2}$ solar cells," Thin Solid Films, vol. 403-404, pp. 280-286, 2002.

[7] S. Hegedus, D. Desai, D. Ryan, and B. McCandless, "Transient degradation and recovery of CdS/CdTe solar cells," in Photovoltaic Specialists Conference, 2005. Conference Record of the Thirty-first IEEE, Jan 2005, pp. 319-322.

[8] R. Sasala and J. Sites, "Time dependent voltage in cuinse sub $_{i} 2 i /$ sub $_{i}$ and cdte solar cells," in Photovoltaic Specialists Conference, 1993., Conference Record of the Twenty Third IEEE. IEEE, 1993, pp. 543-548.

[9] D. Dirnberger, "Uncertainty in PV module measurement-Part II: Verification of rated power and stability problems," Photovoltaics, IEEE Journal of, vol. 4, no. 3, pp. 991-1007, May 2014. 